\title{
Distinguishing trivial and topological zero-energy states in long nanowire junctions
}

\author{
Jorge Cayao and Annica M. Black-Schaffer $\odot$ \\ Department of Physics and Astronomy, Uppsala University, Box 516, S-751 20 Uppsala, Sweden
}

(Received 24 November 2020; revised 17 June 2021; accepted 22 June 2021; published 1 July 2021)

\begin{abstract}
The emergence of zero-energy states in nontopological superconductors represents an inevitable problem that obscures the proper identification of zero-energy Majorana bound states (MBSs) and prevents their use as topologically protected qubits. In this Research Letter we investigate long superconductor-normal-superconductor junctions where trivial zero-energy states, robust over a large range of parameters, appear as a result of helicity and confinement in the normal region. We demonstrate that both equilibrium supercurrents and critical currents are sensitive to variations in the length of the superconductor regions in the topological phase hosting MBSs but, remarkably, no such length dependence exists when robust, but trivial, zero-energy states are present. This strikingly different response originates from the nonlocal nature of the MBSs, and we therefore propose it as a simple protocol for distinguishing between trivial and topological zero-energy states.
\end{abstract}

DOI: 10.1103/PhysRevB.104.L020501

The realization of topological superconductivity and Majorana bound states (MBSs) has generated a great deal of attention in physics, not only because MBSs represent a new superconducting state but also due to their potential for applications [1-6]. Among the most striking properties of MBSs is their nonlocality and associated non-Abelian statistics, crucial for applications in topological quantum computation [7-12]. This has led to a series of experiments using nanowires with spin-orbit coupling (SOC) and proximity-induced $s$-wave superconductivity, where a large enough external magnetic field is predicted to drive the system into a topological phase with MBSs at the wire end points $[13,14]$. So far, the primary detection scheme of MBSs has been a zero-bias conductance peak of height $2 e^{2} / h$ at zero temperature [15-17] due to their unique charge-neutral nature, with several recent experimental results in apparent agreement [18-25].

Recent studies have reported, however, that the observed zero-bias peaks might instead have a mundane trivial origin and hence do not prove the presence of MBSs, as originally thought. In fact, it has been shown that Andreev bound states (ABSs) can emerge at zero energy for magnetic fields well below the topological transition [26-45], with properties similar to those of MBSs. While some such trivial zero-energy states (ZESs) are easily removable by varying system parameters, other trivial ZESs remain robust for a large range of parameters, making the identification of MBSs extremely challenging.

An experimentally relevant regime with highly robust, but trivial, ZESs is found in superconductor-normal-

Published by the American Physical Society under the terms of the Creative Commons Attribution 4.0 International license. Further distribution of this work must maintain attribution to the author(s) and the published article's title, journal citation, and DOI. Funded by Bibsam.
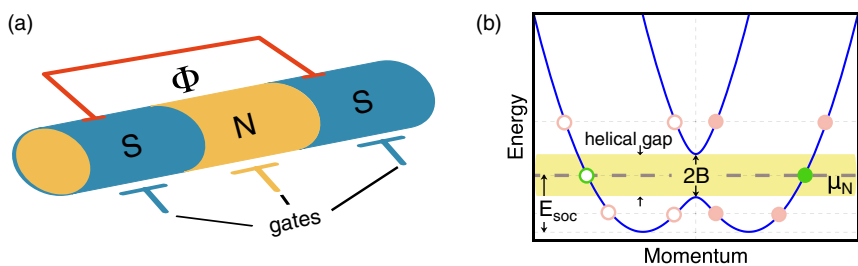

FIG. 1. (a) Semiconducting nanowire with SOC covered by $s$ wave superconductors ( $\mathrm{S}$, blue) leaving an uncovered central region in the normal ( $\mathrm{N}$, yellow) state. $\mathrm{S}$ regions are connected by a grounded superconducting loop allowing control of the phase difference $\phi$ via the flux $\Phi$. Gate voltages individually control the chemical potential in each region. (b) Energy dispersion in the normal state with an applied perpendicular magnetic field producing a gap of size $2 B$ (yellow) and centered around $\mu_{\mathrm{N}}=0$. Inside this gap the system exhibits only two Fermi points, representing counterpropagating states with different spins (solid and open green circles), and this is thus a helical gap. For $\mu$ above or below this gap there are four Fermi points (pink circles). Visibility of the helical gap is lost when $B \gg E_{\mathrm{SOC}}$, with $E_{\mathrm{SOC}}$ being the SOC energy.

superconductor (SNS) junctions with long $\mathrm{N}$ regions, due to confinement and helicity in the normal region $\mathrm{N}$ $[27,28,28,29]$. We stress that typical experimental nanowire setups are likely to belong to this type of junction, as confinement effects appear when contacting a conductor between, e.g., $\mathrm{S}$ leads, as in Fig. 1(a), and also when gating the $\mathrm{N}$ region [46]. Helicity is present whenever the $\mathrm{N}$ region is gated such that its chemical potential lies within the so-called helical energy gap, opened due to a combined effect of SOC and magnetic field; see Fig. 1(b). This helical regime is found at low magnetic fields and thus occurs as an inevitable interim phase before the topological phase at higher fields, which further complicates detecting MBSs. Despite recent efforts [47-66] and its large practical importance, unambiguous experimental signatures distinguishing between robust trivial 
ZESs in the helical regime and topological MBSs are still elusive.

In this Research Letter we show how equilibrium phasebiased transport in long SNS junctions based on nanowires with SOC [see Fig. 1(a)] can clearly distinguish between robust trivial ZESs and topological MBSs. In particular, we design a test of nonlocality, unique to MBSs, using supercurrents and critical currents by varying the lengths of the superconducting $\mathrm{S}$ regions. We demonstrate that these quantities are not affected by the $S$ region lengths when trivial ZESs are present but exhibit strong changes when MBSs emerge. This different behavior occurs due to the Majorana nonlocality and thus provides a distinctive, yet experimentally easily accessible, way to separate topological MBSs from robust trivial ZESs by means of the Josephson effect.

Nanowire junction. We consider long SNS junctions based on a single-channel nanowire with Rashba SOC, using a continuum Hamiltonian given by

$$
H=\left(\frac{p_{x}^{2}}{2 m}-\mu\right) \tau_{z}+\frac{\alpha_{\mathrm{R}}}{\hbar} p_{x} \sigma_{y} \tau_{z}+B \sigma_{x} \tau_{z}+\Delta(x) \sigma_{y} \tau_{y},
$$

where $p_{x}=-i \hbar \partial_{x}$ is the momentum operator, $m$ is the effective electron mass, $\mu(x)$ is the chemical potential dependent on position $x, \alpha_{\mathrm{R}}$ is the Rashba SOC strength, $B$ is the Zeeman field resulting from an external magnetic field, $\Delta(x)$ is the induced $s$-wave superconducting order parameter, and $\sigma_{i}$ and $\tau_{i}$ are the $i$-Pauli matrices in spin and electron-hole spaces, respectively. In the normal state dispersion we find a gap of size $2 B$ at zero momentum and centered around $\mu_{\mathrm{N}}=0$; see Fig. 1(b). For $\mu_{\mathrm{N}}$ within this gap the system exhibits only two Fermi points (green circles), representing counterpropagating states with different spins [67], thus generating a helical phase. Below, we show that this helical phase is particularly interesting as it allows the junction to host robust, but trivial, ZESs.

In order to model finite systems, we discretize the continuum Hamiltonian in Eq. (1) into a tight-binding lattice with spacing $a=10 \mathrm{~nm}$ [68]. This discretized model is then divided into three regions of finite length; see Fig. 1(a): The left and right $\mathrm{S}$ regions host a finite superconducting order parameter $\Delta$, with a finite phase difference $\phi$, while the central $\mathrm{N}$ region is left with $\Delta=0$, giving rise to a finite SNS junction. Each region has length $L_{\mathrm{N}, \mathrm{S}}$ and finite chemical potential $\mu_{\mathrm{N}, \mathrm{S}}$, tunable by means of voltage gates. We study SNS junctions with long $\mathrm{N}$ regions, $L_{\mathrm{N}}=2000 \mathrm{~nm}$, but systematically vary the length of the $S$ regions to differentiate between trivial ZESs and MBSs. We further consider realistic system parameters: $\alpha_{\mathrm{R}}=40 \mathrm{meV} \mathrm{nm}$ and $\Delta=0.5 \mathrm{meV}$, which are in the range of experimental values reported for InSb and InAs nanowires and $\mathrm{Nb}$ and $\mathrm{Al}$ superconductors [2].

Trivial ZESs and topological MBSs. To show the emergence of robust trivial ZESs and also contrast them with the topological MBSs, we first study the low-energy spectrum of long SNS junctions in Fig. 2. In Figs. 2(a) and 2(b) we present the Zeeman-dependent low-energy spectrum at $\phi=0$ for short and long $S$ regions, using $L_{S} \leqslant 2 \ell_{M}$ and $L_{S} \geqslant 2 \ell_{M}$, respectively, where $\ell_{\mathrm{M}}$ is the Majorana localization length. At $B=0$, many low-energy levels appear within the $S$ bulk energy gap $\Delta_{\text {bulk }}$ as a result of having a long $\mathrm{N}$ region. These levels split at finite $B$, and the energy spectrum undergoes two
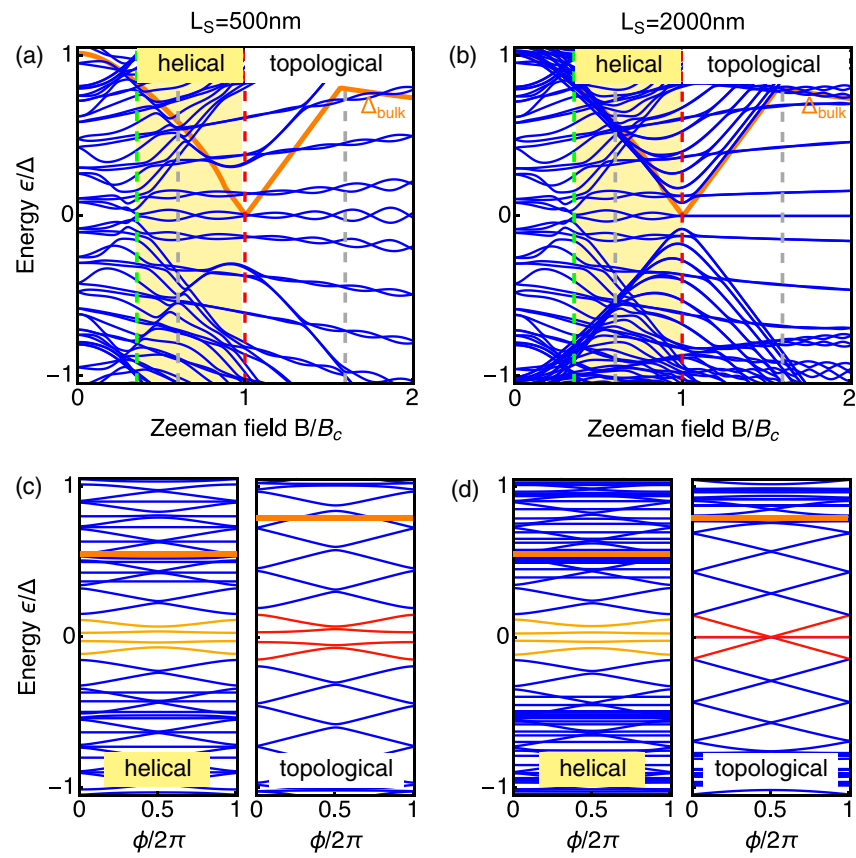

(d)
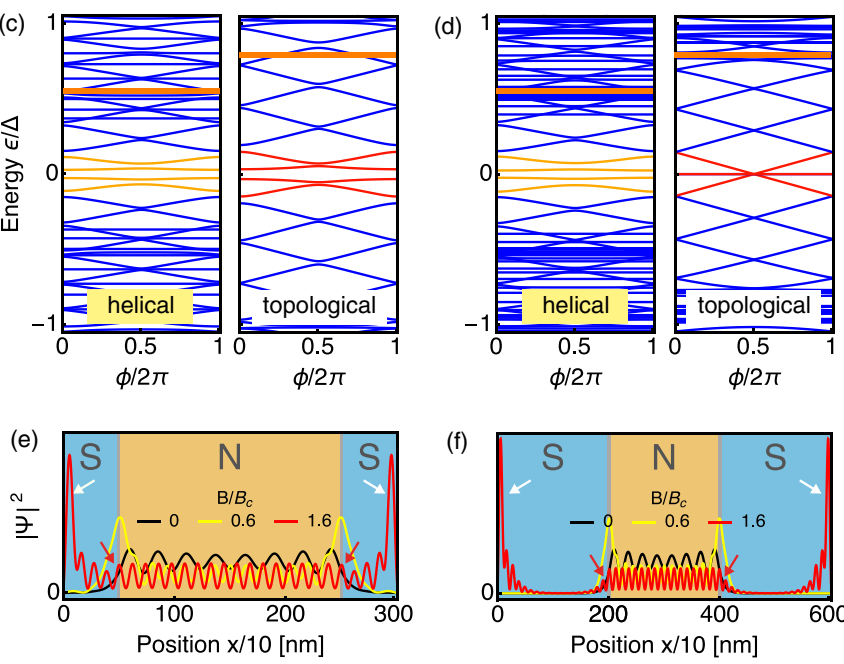

FIG. 2. (a) and (b) Low-energy spectrum in a long SNS junction as a function of Zeeman field $B$ at $\phi=0$ for $L_{S}=500 \mathrm{~nm}$ (a) and $L_{\mathrm{S}}=2000 \mathrm{~nm}$ (b). Vertical green and red dashed lines mark when the $\mathrm{N}$ region becomes helical (yellow region), $B=\mu_{\mathrm{N}}$, and the $\mathrm{S}$ regions become topological, $B=B_{\mathrm{c}}$, respectively. The orange curve corresponds to the minimum $S$ bulk energy gap $\Delta_{\text {bulk }}$. (c) and (d) Phase-dependent low-energy spectrum taken at $B$ in the helical and topological regimes [vertical gray dashed lines in (a) and (b)] for both junctions with the lowest four levels color coded and the thick orange line indicating $\Delta_{\text {bulk }}$. (e) and (f) Spatial dependence of the wave-function amplitude $|\Psi|^{2}$ for the lowest four states [color coded in (c) and (d)] at $\phi=\pi$ at $B=0$ (black curve), in the helical phase (yellow curve), and in the topological phase (red curve). Arrows indicate the outer (white arrows) and inner (red arrows) MBSs. Here, $\mu_{\mathrm{S}}=0.5 \mathrm{meV}, \mu_{\mathrm{N}}=0.25 \mathrm{meV}$.

transitions when $B$ is further increased, reflecting the nature of the $\mathrm{N}$ and $\mathrm{S}$ regions. First, at $B=\mu_{\mathrm{N}}$ (vertical dashed green line) the low-energy spectrum in the $\mathrm{N}$ region exhibits a gapinversion-like feature, which leads to energy levels around zero energy developing parity crossings that oscillate and, remarkably, persist as $B$ increases. This regime occurs as a result of confinement and helicity of the $\mathrm{N}$ region in long SNS junctions. The developed ZESs are topologically trivial and emerge due to helical Fabry-Perot resonances in the $\mathrm{N}$ region [28]. These resonances reflect an instance of helical transport [28,69-73] present when the $\mathrm{N}$ region hosts only spin-polarized counterpropagating states in the helical phase [67]. We verify that these trivial ZESs start to form already for $L_{\mathrm{N}}>250 \mathrm{~nm}$ and are thus expected to be achieved in current experiments; see, e.g., Ref. [41]. These trivial ZESs 
are remarkably robust against parameter changes, as long as the helical phase persists in $\mathrm{N}$.

Second, as $B$ further increases, the $\mathrm{S}$ regions enter into the topological phase at $B=B_{\mathrm{c}}$ [vertical red dashed line in Figs. 2(a) and (b)]. At this point, an energy gap inversion is expected in the spectrum, but due to finite lengths a complete gap closing is not present [47]. We still indicate the bulk gap inversion obtained from Eq. (1) with $\Delta_{\text {bulk }}$ (thick orange curve). In the topological phase, $B>B_{\mathrm{c}}$, two states emerge around zero energy and oscillate with $B$ due to the short length of S [27,74-76], particularly seen in Fig. 2(a). These states are the outer MBSs, located at the outer ends of the SNS junction. The would-be MBSs at the inner ends of the $\mathrm{S}$ regions hybridize strongly across the $\mathrm{N}$ region and are not present unless $\phi=\pi[68,77]$. Note that these outer MBSs oscillate in a very similar fashion to the trivial ZESs in the helical phase, which complicates the distinction of states around zero energy. However, when the $\mathrm{S}$ regions become longer, the oscillations in the topological phase are reduced [see Fig. 2(b), where $L_{S} \geqslant 2 \ell_{\mathrm{M}}$ ], while the behavior in the helical phase is essentially unresponsive to variations in $L_{S}$.

To gain further evidence of the difference between trivial ZESs and topological MBSs, we analyze in Figs. 2(c) and 2(d) the low-energy phase-dependent spectrum at the $B$ fields in the helical and topological regimes marked by the vertical gray dashed lines in Figs. 2(a) and 2(b). First, in both regimes we observe that the multiple energy levels emerging within the gap disperse linearly with $\phi$, an effect attributed to the long-junction nature [68,78-80]. Here, we distinguish the lowest four levels, shown in orange and red for the trivial and topological phases, respectively, from the higher levels also present within the bulk gap $\Delta_{\text {bulk }}$, as well as the quasicontinuum above $\Delta_{\text {bulk }}$. For short $\mathrm{S}$ regions the phase-dependent low-energy spectra in the helical and topological phases are very similar. This indicates that it is extremely challenging in this regime to identify whether the low-energy spectrum, and its associated ZESs, corresponds to a helical trivial or topological phase, as also argued in Ref. [28]. It is, however, possible to find a prominent difference between the helical and topological phases by noting that the lowest four energy levels exhibit a distinct response to an increase in $L_{S}$. This is illustrated in Fig. 2(d), where we clearly see that, as a result of longer $\mathrm{S}$ regions, the splitting around zero energy is reduced in the topological phase, while the helical phase spectrum is almost unchanged. This behavior can be understood by looking at the total wave-function amplitude of the four lowest states in Figs. 2(e) and 2(f). In the topological phase at $\phi=\pi$ the four lowest levels shown in red in Figs. 2(c) and 2(d) represent four MBSs (red curves): The lowest two levels are the two outer MBSs (white arrows), while the next two levels are the two inner MBSs present at $\phi=\pi$ only (red arrows) [68,77,81-83]. We see that for short $L_{\mathrm{S}} \leqslant \ell_{\mathrm{M}}$, the MBS wave functions exhibit a very strong spatial overlap across the $S$ region, which results in finite zero-energy splitting at $\phi=\pi$. For long $S$ with $L_{S} \geqslant \ell_{M}$, the spatial overlap is considerably reduced, and so is the energy splitting at $\phi=\pi$. In contrast, in the helical regime, the lowest states (yellow curves) are always mainly located at the SN and NS interfaces and do not exhibit a noticeable change with the length of $\mathrm{S}$. Note that these helical states are different from the states
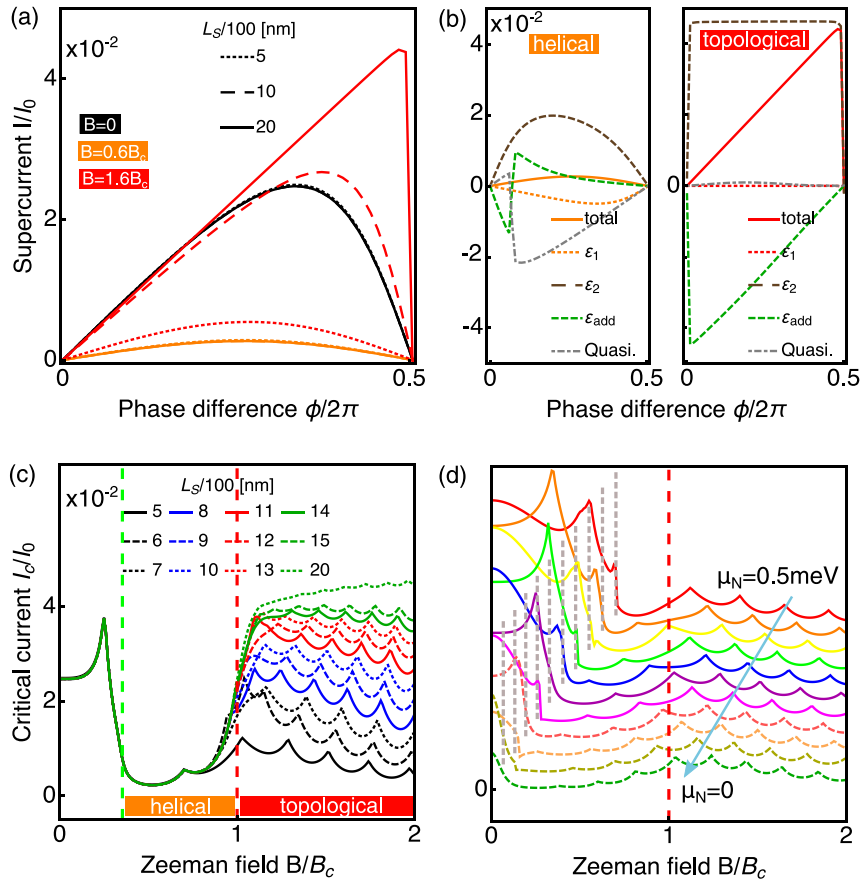

FIG. 3. (a) Current-phase relationship for a long SNS junction in $B=0$ (black curves), helical (orange curves), and topological (red curves) phases and for different $\mathrm{S}$ region lengths $L_{\mathrm{S}}$. Here, $I_{0}=(e \Delta / \hbar)$. (b) Individual contributions to $I(\phi)$ from the first level $\varepsilon_{1}$, the second level $\varepsilon_{2}$, additional levels below $\Delta_{\text {bulk }}\left(\varepsilon_{\text {add }}\right)$, and the quasicontinuum (Quasi.; levels above $\Delta_{\text {bulk }}$ ) for $L_{\mathrm{S}}=2000 \mathrm{~nm}$. (c) and (d) Critical current as a function of the Zeeman field $B$ for the same long junction as in (a) for different $L_{\mathrm{S}}$ (c) and different $\mu_{\mathrm{N}}$ for $L_{\mathrm{S}}=500 \mathrm{~nm}$ (d). Curves in (d) are offset by a constant value for visualization. Vertical gray dashed lines in (d) mark the helical phase transition. The rest of the parameters are as in Fig. 2.

at $B=0$, which are fully extended in the whole $\mathrm{N}$ region (black curves).

The suppression of the energy splitting at $\phi=\pi$ in the topological phase occurring due to the reduction in the spatial overlap between the MBS wave functions in the $S$ regions can directly be interpreted as a result of the nonlocal nature of MBSs. It is thus the MBS nonlocality that causes the different behavior between the trivial helical and topological regimes. Therefore, by measuring the phase-dependent spectrum in SNS junctions, e.g., by spectroscopic means as in Refs. [84-90], it should be possible to identify the nonlocal nature of the topological MBSs and rule out any trivial ZES interpretation.

Current-phase relationship. In order to provide a more distinctive tool to distinguish trivial ZESs from topological MBSs than the Andreev spectrum discussed above, we study the phase-dependent supercurrents. At zero temperature, the current is obtained as $I(\phi)=-(e / \hbar) \sum_{\varepsilon_{n}>0}\left[d \varepsilon_{n}(\phi) / d \phi\right]$, where $\varepsilon_{n}(\phi)$ are the phase-dependent energy levels studied in the previous section. Since our system is of finite length, $I(\phi)$ automatically also includes the contribution of the discrete phase-dependent quasicontinuum [68]. The phase-dependent supercurrents $I(\phi)$ are presented in Fig. 3(a) for different values of the $S$ region length $L_{S}$ and Zeeman field $B$. For a complete understanding, in Fig. 3(b) we also plot the 
individual contribution of the energy levels to $I(\phi)$, separated into the first two levels $\varepsilon_{1,2}$ [shown in orange and red in Figs. 2(c) and 2(d)]; additional levels below $\Delta_{\text {bulk }}$, denoted here as $\varepsilon_{\text {add }}$; and the quasicontinuum above $\Delta_{\text {bulk }}$.

At $B=0, I(\phi)$ exhibits the usual sinelike form, and its behavior does not change when increasing $L_{\mathrm{S}}$, as depicted by the black curves in Fig. 3(a). At finite $B$ in the helical regime, the amplitude of $I(\phi)$ is reduced due to Zeeman depairing, but the overall sinelike profile of $I(\phi)$ and its independence on $L_{\mathrm{S}}$, remarkably, remain; see orange curves. The insensitivity of $I(\phi)$ to $L_{\mathrm{S}}$ for $B<B_{\mathrm{c}}$ is the usual situation in conventional trivial ballistic junctions [91], and here we find that this also applies to junctions hosting trivial ZESs in the helical phase. Note that most states contribute to the current profile; see Fig. 3(b).

The behavior is very different in the topological phase $B>B_{\mathrm{c}}$. Here, $I(\phi)$ instead exhibits a very strong dependence on $L_{\mathrm{S}}$; see red curves in Fig. 3(a). In junctions with short $\mathrm{S}$ regions, $I(\phi)$ has a sinelike behavior and thus is very similar to the trivial helical regime, making them nearly impossible to distinguish. However, when $L_{\mathrm{S}}$ increases, $I(\phi)$ acquires larger amplitudes in the topological phase giving rise to very different current-phase relationships, in contrast to the superimposed curves in the helical regime; $I(\phi)$ also exhibits a notably more steep profile at $\phi=\pi$ that evolves into a sawtooth profile for very long $\mathrm{S}$ regions. This behavior can be understood by inspecting the phase-dependent low-energy spectrum in Figs. 2(c) and 2(d), where we observe that the finite zero-energy splitting at $\phi=\pi$ is considerably reduced for large $L_{\mathrm{S}}$, stemming from the reduced spatial overlap of the outer and inner MBSs' wave functions. This view is further supported by noting that it is the contribution from the second level, $\varepsilon_{2}$, corresponding to the inner MBSs, that largely determines the fast-sign-change profile of $I(\phi)$ at $\phi=\pi$; see Fig. 3(b). In contrast, close to $\phi=0$, this contribution is largely canceled by the additional levels within the bulk gap. Contributions from the quasicontinuum and lowest energy level $\varepsilon_{1}$ (outer MBSs) are both negligibly small, in contrast to the helical regime. The fast sign change in $I(\phi)$ around $\phi=\pi$ thus results from the nonlocal nature of MBSs, which becomes a dominant feature with increasing length of the $\mathrm{S}$ region. Therefore the $L_{\mathrm{S}}$ dependence in the current-phase relationship offers a remarkably distinctive tool to identify MBSs and trivial ZESs, which clearly works even when the sawtooth profile smoothens.

Critical currents. An even simpler experimental tool to distinguish trivial ZESs from MBSs can be obtained by studying the critical current $I_{\mathrm{c}}$, which is the maximum supercurrent that can flow through the junction, $I_{\mathrm{c}}=\max _{\phi}[I(\phi)][77,91]$. In Figs. 3(c) and 3(d) we present the Zeeman-field-dependent critical currents for different $L_{\mathrm{S}}$ and different $\mu_{\mathrm{N}}$, respectively.

As $B$ increases, the critical current undergoes a large change at $B=\mu_{\mathrm{N}}$ when the $\mathrm{N}$ region becomes helical [vertical green dashed line in Fig. 3(c)]. As $B$ further increases within the helical regime, $I_{\mathrm{c}}$ reflects the oscillations with $B$ of the lowest energy levels, seen in Figs. 2(a) and 2(b), but the critical current is notably completely independent of variations in $L_{\mathrm{S}}$. At $B=B_{\mathrm{c}}$ the system enters the topological phase [vertical red dashed line in Figs. 3(c) and 3(d)], but this transition is largely unnoticed in $I_{\mathrm{c}}$, an effect due to contributions from additional levels in the long $\mathrm{N}$ regions, in stark contrast to the case of junctions with very short $L_{\mathrm{N}}[77,92,93]$. In the topological phase, $I_{\mathrm{c}}$ exhibits notable oscillations as a function of $B$ for all shorter $\mathrm{S}$ lengths, due to the zero-energy splitting at $\phi=\pi$. As $L_{\mathrm{S}}$ increases, $I_{\mathrm{c}}$ acquires larger values and the oscillations wash out, consistent with the vanishing of the zero-energy splitting at $\phi=\pi$. The strong dependence of $I_{\mathrm{c}}$ on $L_{\mathrm{S}}$ in the topological regime is a direct consequence of the nonlocal nature of MBSs, which provides unique information even if the oscillations themselves are small. This stands in stark contrast to the insensitivity of $I_{\mathrm{c}}$ to variations in $L_{\mathrm{S}}$ in the topologically trivial helical regime.

We further investigate the strong feature of $I_{\mathrm{c}}$ at the helical transition at $B=\mu_{\mathrm{N}}$ by depleting carriers in $N$, as presented in Fig. 3(d) for a junction with short $\mathrm{S}$ regions (curves offset for visualization). When the chemical potentials in $\mathrm{N}$ and $\mathrm{S}$ are equal (uppermost red curve), $I_{\mathrm{c}}$ is strongly reduced at the helical transition $B=\mu_{\mathrm{N}}$ (vertical gray dashed line). As $\mu_{\mathrm{N}}$ is reduced, the helical transition moves to lower $B$, but it remains a prominent feature in $I_{\mathrm{c}}$. For all these different junctions there is no notable evidence of the topological phase transition (vertical red dashed line) in $I_{\mathrm{c}}$, which indicates that it is nearly impossible to know whether the system is in the topological phase from a single measurement. However, as we established in Fig. 3(c), the topological phase is clearly detectable when probing the critical current as a function of length of the $S$ regions. We further confirm that for longer $\mathrm{S}$ regions, only the oscillations arising from MBSs are washed out, while the signature of the helical transition and the oscillations due to the helical ZESs remain robust (not shown).

We have verified that the above-reported findings are robust against scalar disorder (of the order of $\mu_{\mathrm{S}}$ ), against reduction in normal transmission at the SN interfaces, and also against finite temperatures (below the superconducting transition temperature). Moreover, we have also studied an alternative approach to test nonlocality where we keep $L_{S}$ fixed, with all our findings presented here remaining intact (see the Supplemental Material [94]). Based on current developments, we also stress that the low-energy spectrum and supercurrents, including critical currents, in SNS junctions, have been measured in junctions similar to the ones studied in this Research Letter [41,90,95-98], and thus our findings provide an achievable route to distinguish trivial ZESs and topological MBSs.

In conclusion, we have demonstrated that robust trivial zero-energy states and topological zero-energy Majorana bound states, both emerging in long SNS junctions, can be clearly distinguished by means of equilibrium phase-biased transport in junctions with varying lengths of the $S$ regions. These transport signatures are due to the unique nonlocality of the Majorana bound states and can thus be used to distinguish Majorana bound states from trivial zero-energy states, even independent of the origin of the latter.

We thank J. C. Estrada Saldaña and E. Lee for insightful and motivating discussions. We acknowledge financial support from the Swedish Research Council (Vetenskapsrådet Grant No. 2018-03488), the Knut and Alice Wallenberg Foundation through the Wallenberg Academy Fellows program, and the EU-COST Action CA-16218 NANCOHYBRI. 
[1] R. Aguado, Riv. Nuovo Cimento 40, 523 (2017).

[2] R. M. Lutchyn, E. P. Bakkers, L. P. Kouwenhoven, P. Krogstrup, C. M. Marcus, and Y. Oreg, Nat. Rev. Mater. 3, 52 (2018).

[3] H. Zhang, D. E. Liu, M. Wimmer, and L. P. Kouwenhoven, Nat. Commun. 10, 5128 (2019).

[4] C. Beenakker, SciPost Phys. Lect. Notes 15 (2020).

[5] Y. Oreg and F. von Oppen, Annu. Rev. Condens. Matter Phys. 11, 397 (2020).

[6] R. Aguado, Appl. Phys. Lett. 117, 240501 (2020).

[7] A. Y. Kitaev, Phys.-Usp. 44, 131 (2001).

[8] D. A. Ivanov, Phys. Rev. Lett. 86, 268 (2001).

[9] C. Nayak, S. H. Simon, A. Stern, M. Freedman, and S. Das Sarma, Rev. Mod. Phys. 80, 1083 (2008).

[10] S. D. Sarma, M. Freedman, and C. Nayak, npj Quantum Inf. 1, 15001 (2015).

[11] J. Alicea, Y. Oreg, G. Refael, F. Von Oppen, and M. P. Fisher, Nat. Phys. 7, 412 (2011).

[12] D. Aasen, M. Hell, R. V. Mishmash, A. Higginbotham, J. Danon, M. Leijnse, T. S. Jespersen, J. A. Folk, C. M. Marcus, K. Flensberg, and J. Alicea, Phys. Rev. X 6, 031016 (2016).

[13] R. M. Lutchyn, J. D. Sau, and S. Das Sarma, Phys. Rev. Lett. 105, 077001 (2010).

[14] Y. Oreg, G. Refael, and F. von Oppen, Phys. Rev. Lett. 105, 177002 (2010).

[15] C. J. Bolech and E. Demler, Phys. Rev. Lett. 98, 237002 (2007).

[16] K. T. Law, P. A. Lee, and T. K. Ng, Phys. Rev. Lett. 103, 237001 (2009).

[17] K. Flensberg, Phys. Rev. B 82, 180516(R) (2010).

[18] V. Mourik, K. Zuo, S. Frolov, S. Plissard, E. Bakkers, and L. Kouwenhoven, Science 336, 1003 (2012).

[19] A. P. Higginbotham, S. M. Albrecht, G. Kirsanskas, W. Chang, F. Kuemmeth, P. Krogstrup, T. S. J. J. Nygård, K. Flensberg, and C. M. Marcus, Nat. Phys. 11, 1017 (2015).

[20] M. T. Deng, S. Vaitiekenas, E. B. Hansen, J. Danon, M. Leijnse, K. Flensberg, J. Nygård, P. Krogstrup, and C. M. Marcus, Science 354, 1557 (2016).

[21] S. M. Albrecht, A. P. Higginbotham, M. Madsen, F. Kuemmeth, T. S. Jespersen, J. Nygård, P. Krogstrup, and C. M. Marcus, Nature (London) 531, 206 (2016).

[22] H. Zhang, Ö. Gül, S. Conesa-Boj, K. Zuo, V. Mourik, F. K. de Vries, J. van Veen, D. J. van Woerkom, M. P. Nowak, M. Wimmer, D. Car, S. Plissard, E. P. A. M. Bakkers, M. Quintero-Pérez, S. Goswami, K. Watanabe, T. Taniguchi, and L. P. Kouwenhoven, Nat. Commun. 8, 16025 (2017).

[23] H. J. Suominen, M. Kjaergaard, A. R. Hamilton, J. Shabani, C. J. Palmstrøm, C. M. Marcus, and F. Nichele, Phys. Rev. Lett. 119, 176805 (2017).

[24] F. Nichele, A. C. C. Drachmann, A. M. Whiticar, E. C. T. O'Farrell, H. J. Suominen, A. Fornieri, T. Wang, G. C. Gardner, C. Thomas, A. T. Hatke, P. Krogstrup, M. J. Manfra, K. Flensberg, and C. M. Marcus, Phys. Rev. Lett. 119, 136803 (2017).

[25] Ö. Gül, H. Zhang, J. D. S. Bommer, M. W. A. de Moor, D. Car, S. R. Plissard, E. P. A. M. Bakkers, A. Geresdi, K. Watanabe, T. Taniguchi, and L. P. Kouwenhoven, Nat. Nanotechnol. 13, 192 (2018).

[26] G. Kells, D. Meidan, and P. W. Brouwer, Phys. Rev. B 86, 100503(R) (2012).
[27] E. Prada, P. San-Jose, and R. Aguado, Phys. Rev. B 86, 180503(R) (2012).

[28] J. Cayao, E. Prada, P. San-Jose, and R. Aguado, Phys. Rev. B 91, 024514 (2015).

[29] P. San-José, J. Cayao, E. Prada, and R. Aguado, Sci. Rep. 6, 21427 (2016)

[30] C.-X. Liu, J. D. Sau, T. D. Stanescu, and S. Das Sarma, Phys. Rev. B 96, 075161 (2017).

[31] A. Ptok, A. Kobiałka, and T. Domański, Phys. Rev. B 96, 195430 (2017).

[32] C. Fleckenstein, F. Domínguez, N. Traverso Ziani, and B. Trauzettel, Phys. Rev. B 97, 155425 (2018).

[33] C. Reeg, O. Dmytruk, D. Chevallier, D. Loss, and J. Klinovaja, Phys. Rev. B 98, 245407 (2018).

[34] J. Chen, B. D. Woods, P. Yu, M. Hocevar, D. Car, S. R. Plissard, E. P. A. M. Bakkers, T. D. Stanescu, and S. M. Frolov, Phys. Rev. Lett. 123, 107703 (2019).

[35] T. D. Stanescu and S. Tewari, Phys. Rev. B 100, 155429 (2019).

[36] T. Dvir, M. Aprili, C. H. L. Quay, and H. Steinberg, Phys. Rev. Lett. 123, 217003 (2019).

[37] A. Vuik, B. Nijholt, A. R. Akhmerov, and M. Wimmer, SciPost Phys. 7, 61 (2019).

[38] J. Avila, F. Peñaranda, E. Prada, P. San-Jose, and R. Aguado, Commun. Phys. 2, 133 (2019).

[39] H. Pan and S. Das Sarma, Phys. Rev. Research 2, 013377 (2020).

[40] C. Jünger, R. Delagrange, D. Chevallier, S. Lehmann, K. A. Dick, C. Thelander, J. Klinovaja, D. Loss, A. Baumgartner, and C. Schönenberger, Phys. Rev. Lett. 125, 017701 (2020).

[41] D. Razmadze, E. C. T. O'Farrell, P. Krogstrup, and C. M. Marcus, Phys. Rev. Lett. 125, 116803 (2020).

[42] O. Dmytruk, D. Loss, and J. Klinovaja, Phys. Rev. B 102, 245431 (2020).

[43] P. Yu, J. Chen, M. Gomanko, G. Badawy, E. P. A. M. Bakkers, K. Zuo, V. Mourik, and S. Frolov, Nat. Phys. 17, 482 (2021).

[44] M. Valentini, F. Peñaranda, A. Hofmann, M. Brauns, R. Hauschild, P. Krogstrup, P. San-Jose, E. Prada, R. Aguado, and G. Katsaros, arXiv:2008.02348.

[45] E. Prada, P. San-Jose, M. W. de Moor, A. Geresdi, E. J. Lee, J. Klinovaja, D. Loss, J. Nygård, R. Aguado, and L. P. Kouwenhoven, Nat. Rev. Phys. 2 , 575 (2020).

[46] M.-T. Deng, S. Vaitiekenas, E. Prada, P. San-Jose, J. Nygård, P. Krogstrup, R. Aguado, and C. M. Marcus, Phys. Rev. B 98, 085125 (2018).

[47] R. V. Mishmash, D. Aasen, A. P. Higginbotham, and J. Alicea, Phys. Rev. B 93, 245404 (2016).

[48] J. Liu, J. Song, Q.-F. Sun, and X. C. Xie, Phys. Rev. B 96, 195307 (2017).

[49] T. O. Rosdahl, A. Vuik, M. Kjaergaard, and A. R. Akhmerov, Phys. Rev. B 97, 045421 (2018).

[50] C. Schrade and L. Fu, arXiv:1809.06370.

[51] C. Moore, T. D. Stanescu, and S. Tewari, Phys. Rev. B 97, 165302 (2018).

[52] C.-X. Liu, J. D. Sau, and S. Das Sarma, Phys. Rev. B 97, 214502 (2018).

[53] M. Hell, K. Flensberg, and M. Leijnse, Phys. Rev. B 97, 161401(R) (2018).

[54] L. S. Ricco, M. de Souza, M. S. Figueira, I. A. Shelykh, and A. C. Seridonio, Phys. Rev. B 99, 155159 (2019). 
[55] K. Yavilberg, E. Ginossar, and E. Grosfeld, Phys. Rev. B 100, 241408(R) (2019).

[56] O. A. Awoga, J. Cayao, and A. M. Black-Schaffer, Phys. Rev. Lett. 123, 117001 (2019).

[57] J. Schulenborg and K. Flensberg, Phys. Rev. B 101, 014512 (2020).

[58] G. Zhang and C. Spånslätt, Phys. Rev. B 102, 045111 (2020).

[59] A. Schuray, M. Rammler, and P. Recher, Phys. Rev. B 102, 045303 (2020).

[60] A. Grabsch, Y. Cheipesh, and C. W. J. Beenakker, Adv. Quantum Technol. 3, 1900110 (2020).

[61] H. Pan, J. D. Sau, and S. D. Sarma, Phys. Rev. B 103, 014513 (2021).

[62] D. Liu, Z. Cao, X. Liu, H. Zhang, and D. E. Liu, arXiv:2009.09996.

[63] J. Danon, A. B. Hellenes, E. B. Hansen, L. Casparis, A. P. Higginbotham, and K. Flensberg, Phys. Rev. Lett. 124, 036801 (2020).

[64] L. S. Ricco, J. E. Sanches, Y. Marques, M. de Souza, M. S. Figueira, I. A. Shelykh, and A. C. Seridonio, arXiv:2004.14182.

[65] M. Thamm and B. Rosenow, Phys. Rev. Research 3, 023221 (2021).

[66] W. Chen, J. Wang, Y. Wu, J. Liu, and X. C. Xie, arXiv:2005.00735.

[67] P. Středa and P. Šeba, Phys. Rev. Lett. 90, 256601 (2003).

[68] J. Cayao, A. M. Black-Schaffer, E. Prada, and R. Aguado, Beilstein J. Nanotechnol. 9, 1339 (2018).

[69] D. Rainis and D. Loss, Phys. Rev. B 90, 235415 (2014).

[70] J. Kammhuber, M. C. Cassidy, F. Pei, M. P. Nowak, A. Vuik, Ö. Gül, D. Car, S. Plissard, E. Bakkers, M. Wimmer, and L. P. Kouwenhoven, Nat. Commun. 8, 478 (2017).

[71] J. Sun, R. S. Deacon, R. Wang, J. Yao, C. M. Lieber, and K. Ishibashi, Nano Lett. 18, 6144 (2018).

[72] J. C. Estrada Saldaña, Y.-M. Niquet, J.-P. Cleuziou, E. J. H. Lee, D. Car, S. R. Plissard, E. P. A. M. Bakkers, and S. De Franceschi, Nano Lett. 18, 2282 (2018).

[73] J. Sun, R. S. Deacon, X. Liu, J. Yao, and K. Ishibashi, Appl. Phys. Lett. 117, 052403 (2020).

[74] J. Klinovaja and D. Loss, Phys. Rev. B 86, 085408 (2012).

[75] S. Das Sarma, J. D. Sau, and T. D. Stanescu, Phys. Rev. B 86, 220506(R) (2012).

[76] D. Rainis, L. Trifunovic, J. Klinovaja, and D. Loss, Phys. Rev. B 87, 024515 (2013).

[77] J. Cayao, P. San-Jose, A. M. Black-Schaffer, R. Aguado, and E. Prada, Phys. Rev. B 96, 205425 (2017).

[78] A. Zagoskin, Quantum Theory of Many-Body Systems: Techniques and Applications (Springer, New York, 2014).

[79] C. W. J. Beenakker, D. I. Pikulin, T. Hyart, H. Schomerus, and J. P. Dahlhaus, Phys. Rev. Lett. 110, 017003 (2013).

[80] A. Murani, A. Chepelianskii, S. Guéron, and H. Bouchiat, Phys. Rev. B 96, 165415 (2017).

[81] D. I. Pikulin and Y. V. Nazarov, Phys. Rev. B 86, 140504(R) (2012).

[82] P. San-Jose, E. Prada, and R. Aguado, Phys. Rev. Lett. 108, 257001 (2012).
[83] Y. Peng, F. Pientka, E. Berg, Y. Oreg, and F. von Oppen, Phys. Rev. B 94, 085409 (2016).

[84] E. J. H. Lee, X. Jiang, M. Houzet, R. Aguado, C. M. Lieber, and S. D. Franceschi, Nat. Nanotechnol. 9, 79 (2014).

[85] Y.-J. Doh, J. A. van Dam, A. L. Roest, E. P. A. M. Bakkers, L. P. Kouwenhoven, and S. De Franceschi, Science 309, 272 (2005).

[86] J.-D. Pillet, C. H. L. Quay, P. Morfin, C. Bena, A. L. Yeyati, and P. Joyez, Nat. Phys. 6, 965 (2010).

[87] H. A. Nilsson, P. Samuelsson, P. Caroff, and H. Q. Xu, Nano Lett. 12, 228 (2012).

[88] W. Chang, V. E. Manucharyan, T. S. Jespersen, J. Nygård, and C. M. Marcus, Phys. Rev. Lett. 110, 217005 (2013).

[89] M. Hays, G. de Lange, K. Serniak, D. J. van Woerkom, D. Bouman, P. Krogstrup, J. Nygård, A. Geresdi, and M. H. Devoret, Phys. Rev. Lett. 121, 047001 (2018).

[90] L. Tosi, C. Metzger, M. F. Goffman, C. Urbina, H. Pothier, S. Park, A. L. Yeyati, J. Nygård, and P. Krogstrup, Phys. Rev. X 9, 011010 (2019).

[91] C. Beenakker, in Transport Phenomena in Mesoscopic Systems: Proceedings of the 14th Taniguchi Symposium, Shima, Japan, November 10-14, 1991, Springer Series in Solid-State Sciences Vol. 109 (Springer, New York, 1992), p. 235.

[92] P. San-José, J. Cayao, E. Prada, and R. Aguado, New J. Phys. 15, 075019 (2013).

[93] P. San-Jose, E. Prada, and R. Aguado, Phys. Rev. Lett. 112, 137001 (2014).

[94] See Supplemental Material at http://link.aps.org/supplemental/ 10.1103/PhysRevB.104.L020501 for an alternative way to test nonlocality in phase-biased supercurrents at fixed lengths of the superconducting regions, which also includes Refs. [99-101].

[95] J. Tiira, E. Strambini, M. Amado, S. Roddaro, P. San-Jose, R. Aguado, F. S. Bergeret, D. Ercolani, L. Sorba, and F. Giazotto, Nat. Commun. 8, 14984 (2017).

[96] K. Zuo, V. Mourik, D. B. Szombati, B. Nijholt, D. J. van Woerkom, A. Geresdi, J. Chen, V. P. Ostroukh, A. R. Akhmerov, S. R. Plissard, D. Car, E. P. A. M. Bakkers, D. I. Pikulin, L. P. Kouwenhoven, and S. M. Frolov, Phys. Rev. Lett. 119, 187704 (2017).

[97] M. Kjaergaard, H. J. Suominen, M. P. Nowak, A. R. Akhmerov, J. Shabani, C. J. Palmstrøm, F. Nichele, and C. M. Marcus, Phys. Rev. Appl. 7, 034029 (2017).

[98] F. Nichele, E. Portolés, A. Fornieri, A. M. Whiticar, A. C. C. Drachmann, S. Gronin, T. Wang, G. C. Gardner, C. Thomas, A. T. Hatke, M. J. Manfra, and C. M. Marcus, Phys. Rev. Lett. 124, 226801 (2020).

[99] Y. Liu, S. Vaitiekenas, S. Martí-Sánchez, C. Koch, S. Hart, Z. Cui, T. Kanne, S. A. Khan, R. Tanta, S. Upadhyay, M. Espiñeira Cachaza, C. M. Marcus, J. Arbiol, K. A. Moler, and P. Krogstrup, Nano Lett. 20, 456 (2020).

[100] Z. Yang, B. Heischmidt, S. Gazibegovic, G. Badawy, D. Car, P. A. Crowell, E. P. Bakkers, and V. S. Pribiag, Nano Lett. 20, 3232 (2020).

[101] S. Vaitiekėnas, Y. Liu, P. Krogstrup, and C. Marcus, Nat. Phys. 17, 43 (2021). 